\title{
JNJ872 inhibits influenza A virus replication without altering cellular antiviral responses
}

\section{$\mathrm{Fu}, \mathrm{Yu}$}

2016-09

Fu , Y, Gaelings , L , Soderholm , S , Belanov , S , Nandania , J , Nyman , T A , Matikainen , S , Anders , S , Velagapudi , V \& Kainov , D E 2016 , ' JNJ872 inhibits influenza A virus replication without altering cellular antiviral responses ' , Antiviral Research, vol. 133 , pp. 23-31 . https://doi.org/10.1016/j.antiviral.2016.07.008

http://hdl.handle.net/10138/228312

https://doi.org/10.1016/j.antiviral.2016.07.008

publishedVersion

Downloaded from Helda, University of Helsinki institutional repository.

This is an electronic reprint of the original article.

This reprint may differ from the original in pagination and typographic detail.

Please cite the original version. 


\title{
JNJ872 inhibits influenza A virus replication without altering cellular antiviral responses
}

\author{
Yu Fu a , Lana Gaelings a , Sandra Söderholm ${ }^{\text {b, c }}$, Sergei Belanov ${ }^{\text {a }}$, Jatin Nandania a, \\ Tuula A. Nyman ${ }^{\text {b }}$, Sampsa Matikainen ${ }^{\text {c }}$, Simon Anders ${ }^{\text {a }}$, Vidya Velagapudi ${ }^{\text {a }}$, \\ Denis E. Kainov ${ }^{\text {a, * }}$ \\ ${ }^{a}$ The Institute for Molecular Medicine Finland (FIMM), University of Helsinki, Finland \\ ${ }^{\mathrm{b}}$ Institute of Biotechnology (BI), University of Helsinki, Finland \\ ${ }^{\mathrm{c}}$ Finnish Institute of Occupational Health (TTL), Helsinki, Finland
}

\section{A R T I C L E I N F O}

\section{Article history:}

Received 3 May 2016

Received in revised form

8 July 2016

Accepted 11 July 2016

Available online 20 July 2016

\begin{abstract}
A B S T R A C T
JNJ-63623872 (formally known as VX-787; referred to here as JNJ872) is an orally bioavailable compound, which is in phase II clinical trials for the treatment of influenza A virus (IAV) infections. Here we show that JNJ872 inhibits at nanomolar concentrations the transcription of viral RNA in IAV-infected human macrophages by targeting a highly conserved site on the cap-snatching domain of influenza polymerase basic 2 protein (PB2). Furthermore, even lower concentrations of JNJ872 protected macrophages from IAV-mediated death when given in combination with $100 \mathrm{nM}$ gemcitabine, which also attenuated transcription and replication of viral RNA. Importantly, treating human macrophages with JNJ872 allowed expression of many immune-related and other genes, involved in antiviral responses, such as indoleamine 2,3-dioxygenase 1 (IDO), and cytosolic 5'-nucleotidase $3 \mathrm{~A}$ (NT5C3A). Moreover, our targeted metabolomics analysis indicate that treatment with JNJ782 did not interfere with metabolic responses to infection, which further supported our transcriptomics results. Thus, VX-737 alone or in combination with other drugs could be beneficial for treating IAV infected patients, because it would allow the development of antiviral responses and, thereby, protect individuals from current and future infections with closely related IAV strains.
\end{abstract}

() 2016 Elsevier B.V. All rights reserved.

\section{Introduction}

Influenza A viruses (IAVs) mutate rapidly. Rapid evolution allows emerging viruses to overcome the prevailing immunity in the human population and to cause global epidemics and pandemics (Belanov et al., 2015). In order to combat influenza outbreaks several antiviral drugs have been developed (Edinger et al., 2014; Loregian et al., 2014). In particular, amantadine, rimantadine, oseltamivir, zanamivir, laninamivir, peramivir and favipiravir ( $\mathrm{T}$ 705) were approved in USA for treatment of IAV infections. However, almost all IAV strains became resistant to amantadine and rimantadine, due to mutations in viral M2 protein (http://www. influenzareport.com/ir/drugs/amanta.htm). Oseltamivir is widely used for treating seasonal IAV infections; however, oseltamivir-

\footnotetext{
* Corresponding author.

E-mail address: denis.kainov@helsinki.fi (D.E. Kainov).
}

resistant IAV strains emerge and reduce the efficacy of the treatment due to mutations in viral NA protein (Li et al., 2015). Therefore, there is an unmet medical need for newer antivirals to treat influenza.

JNJ872 is an investigational drug, which was designed to target viral PB2. Virus-directed JNJ872 has progressed to phase II clinical trials as an anti-influenza drug candidate (www.clinicaltrials.gov, NCT02342249 and NCT02532283) (Byrn et al., 2015; Clark et al., 2014). Here, we show that JNJ872 not only efficiently inhibited IAV infection of human macrophages, but also allowed the development of innate immune responses, and thus might have minimal side effects in vivo.

\section{Materials and methods}

\subsection{Antiviral agents and other reagents}

JNJ872 and gemcitabine were from Janssen and Selleck 
Chemicals, USA, respectively. Compounds were dissolved in $100 \%$ dimethyl sulfoxide (Sigma-Aldrich) to obtain $10 \mathrm{mM}$ stock solutions.

All the metabolite standards, ammonium formate, ammonium acetate and ammonium hydroxide were obtained from SigmaAldrich (Helsinki, Finland). Formic acid (FA), 2-proponol, acetonitrile (ACN), and methanol (all HiPerSolv CHROMANORM, HPLC grade, BDH Prolabo) were purchased from VWR International (Helsinki, Finland). Isotopically labeled internal standards were obtained from Cambridge Isotope Laboratory Inc., USA (Ordered from Euroiso-Top, France). Deionized Milliq water up to a resistivity of $18 \mathrm{M} \Omega$ was purified with a purification system (Barnstead
EASYpure RoDi ultrapure water purification system, Thermo Scientific, Ohio, USA).

\subsection{Cells and viruses}

Human primary macrophages were derived from leukocyte-rich buffy coats from healthy blood donors (Finnish Red Cross Blood Transfusion Service, Helsinki, Finland). Monocytes were isolated and differentiated into macrophages as described previously (Pirhonen et al., 1999). Monocytes were seeded in 96- or 6-well plates and cultured in serum free macrophage media (Gibco) supplemented with $10 \mathrm{ng} / \mathrm{ml}$ granulocyte macrophage colony
A

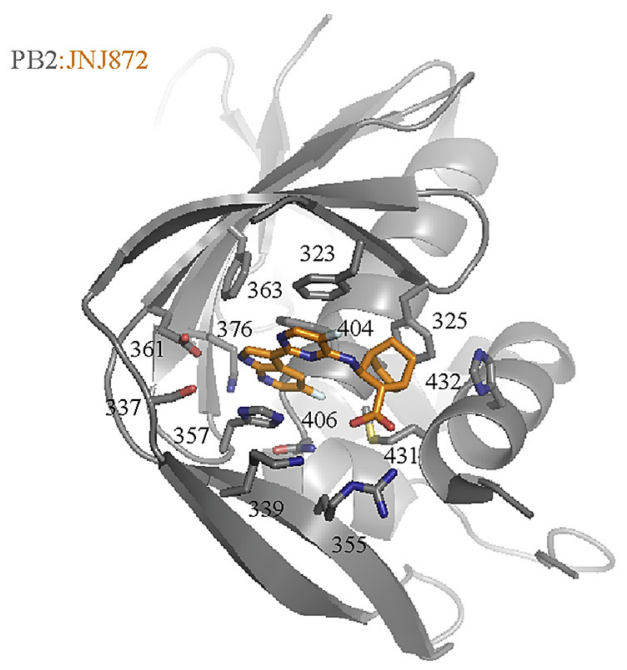

$\mathrm{C}$

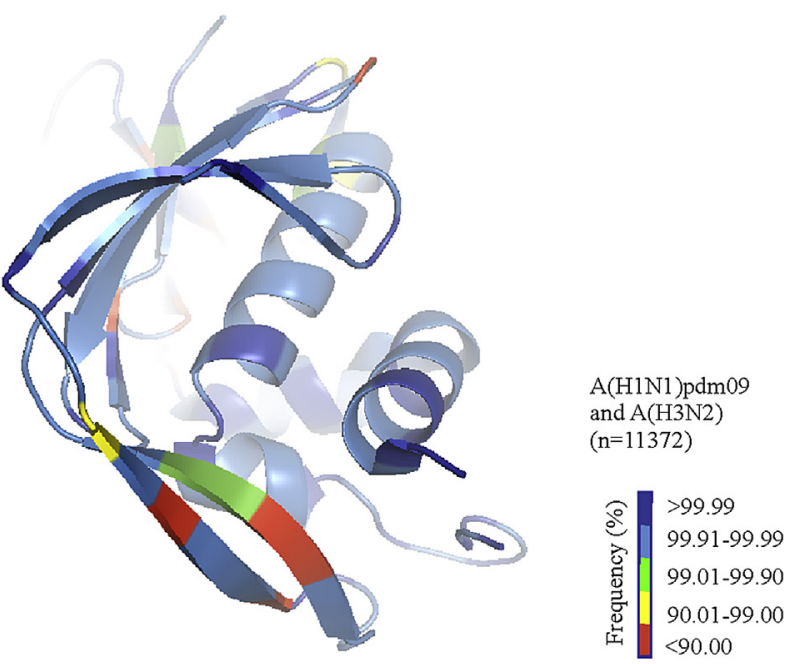

B
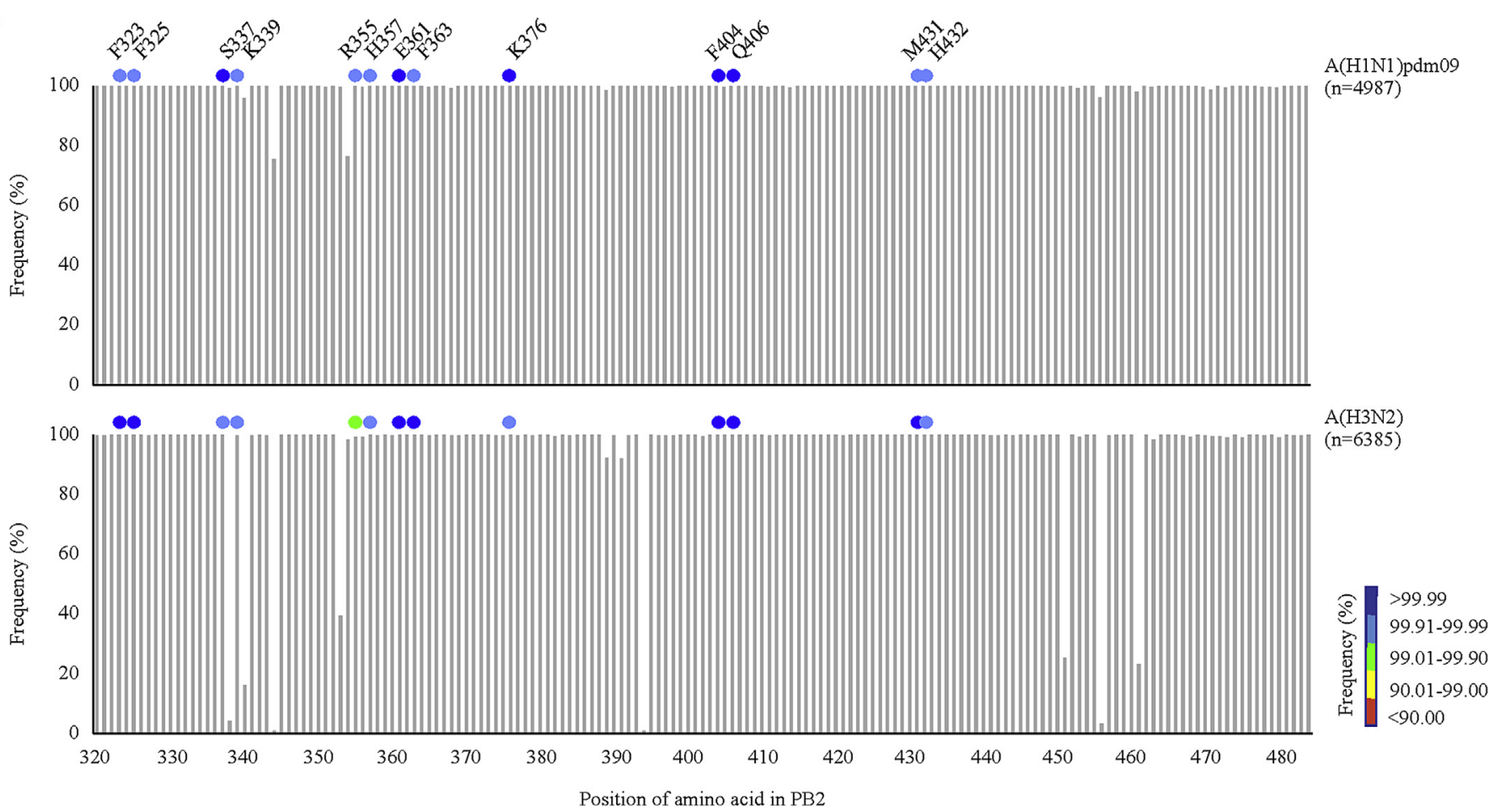

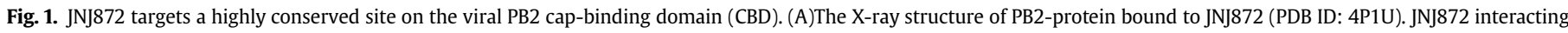

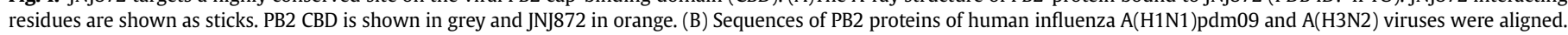

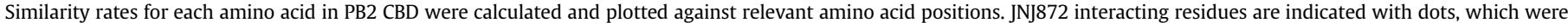

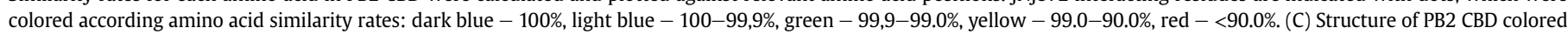
according to similarity rates. 
A

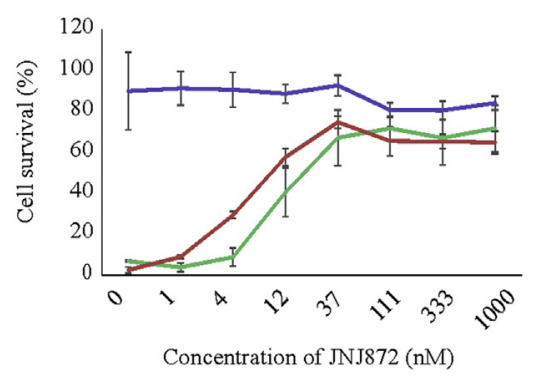

B

\begin{tabular}{l|cccl}
$\begin{array}{l}\text { IAV } \\
\text { strain }\end{array}$ & $\begin{array}{r}\text { EC90 } \\
(\mathbf{n M})\end{array}$ & $\begin{array}{r}\text { EC50 } \\
(\mathbf{n M})\end{array}$ & $\begin{array}{l}\text { CC50 } \\
(\mathbf{n M})\end{array}$ & $\begin{array}{l}\text { SI } \\
\text { (CC50/EC50) }\end{array}$ \\
\hline $\mathrm{A}(\mathrm{H} 1 \mathrm{~N} 1)$ & $30 \pm 3$ & $8 \pm 2$ & $>1000$ & $>125$ \\
$\mathrm{~A}(\mathrm{H} 3 \mathrm{~N} 2)$ & $20 \pm 1$ & $12 \pm 1$ & $>1000$ & $>83$ \\
\hline
\end{tabular}

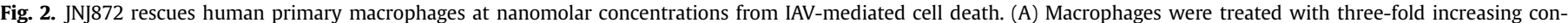

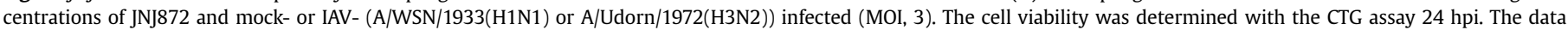

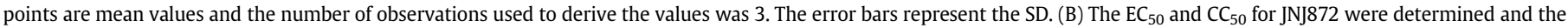
respective SI values were calculated. The data points are mean values and the number of observations used to derive the values was 3 .

stimulating factor (GM-CSF; Biosource International) and $50 \mathrm{U} / \mathrm{mL}$ penicillin-streptomycin (Lonza) at $37{ }^{\circ} \mathrm{C}$ and $5 \% \mathrm{CO}_{2}$ for 7 days, polarizing the monocytes into macrophages of the acute proinflammatory M1-phenotype. Before stimulation, the media was replaced with fresh GM-CSF free macrophage media and macrophages were infected with IAVs.

Human influenza A/WSN/33(H1N1) virus was generated using the eight-plasmid-based reverse genetics system in HEK293T and Vero cells as described previously (Anastasina et al., 2015; Hoffmann et al., 2000).

Human pathogenic influenza A/Udorn/307/1972 (H3N2), provided by the National Institute for Health and Welfare, was cultured in embryonated hen eggs and the virus stock, with a titer of 256 hemagglutination $\mathrm{U} / \mathrm{ml}$, was stored at $-80^{\circ} \mathrm{C}$. A virus dose of 2.56 hemagglutination $\mathrm{U} / \mathrm{ml}$ was used in the infection experiments unless stated otherwise. The virus experiments were carried out under BSL-2 conditions and in compliance with regulations of the University of Helsinki (permit No 21/M/09). Viruses were titered in MDCK cells using a plaque assay as described previously (Denisova et al., 2012).

\subsection{Cell viability assay}

The compound cytotoxicity and efficacy testing was performed in 96-well plates with macrophages at 95\% confluence. The compounds were added to the medium, and 30 min later, the cells were infected with virus or non-infected (mock). The cell viability was analyzed with the Cell Titer Glo assay (CTG; Promega) at $24 \mathrm{hpi}$. The luminescence was read with a PHERAstar FS plate reader (BMG Labtech).

The $50 \%$ cytotoxic concentration $\left(\mathrm{CC}_{50}\right)$ of JNJ872 treatment was determined with the CTG assay in macrophages treated with different concentrations of the compounds $(0-1000 \mathrm{nM})$ for $24 \mathrm{~h}$. The antiviral effect of JNJ872, the half maximal effective concentration $\left(\mathrm{EC}_{50}\right)$, i.e. the ability of the compound to reduce the virus production to $50 \%$, was calculated by measuring the titers of viruses grown in cells (initial moi 0.1 ) for $24 \mathrm{~h}$ in the presence of 0-1000 nM JNJ872 as described (Denisova et al., 2014). The selectivity index (SI) was defined as the $\mathrm{CC}_{50} / \mathrm{EC}_{50}$ ratio.

In the drug combination experiment, macrophages were treated with a constant concentration of one drug $\left(\mathrm{EC}_{10}\right)$ and increasing concentrations of another. Viability of mock and IAV infected cells was measured by using the CTG assay. The minimal concentrations of JNJ872 which in combination with $100 \mathrm{nM}$ gemcitabine efficiently rescued $50 \%$ of infected cells were calculated.

\subsection{Reverse-transcription quantitative polymerase chain reaction $(R T-q P C R)$}

RT-qPCR was performed with the ABI PRISM 7500 Sequence Detection System (Applied Biosystems) using Fast SYBR Green Master Mix (Applied Biosystems). IAV M1 (forward primer: 5'GACCRATCCTGTCACCTCTGAC-3' and reverse primer: 5'-AGGGCATTYTGGACAAAKCGTCTA-3', Sigma Aldrich) (http://www.who. int/csr/resources/publications/swineflu/realtimeptpcr/en/index. html) and cellular GAPDH (forward primer: 5'GGCTGGGGCTCCATTTGCAGGG-3' and reverse primer: $5^{\prime}$ TGACCTTGGCCAGGGGTGCT-3' from Oligomer Oy) were analyzed. The relative gene expression differences were calculated as described before (Ohman et al., 2010) and the results are represented as relative units (RU). Technical triplicates of each sample were performed on the same qPCR plate and non-templates and non-reverse transcriptase samples were analyzed as negative controls. Statistical significance ( $p<0.05)$ of the quantitation results was evaluated with Student $t$-test.

\subsection{Gene expression profiling}

RNA was extracted from IAV- or mock-infected macrophages $8 \mathrm{~h}$ post infection using RNeasy Plus mini kit (Qiagen). Gene expression profiling was performed as described previously (Denisova et al., 2014) using expression microarrays (Illumina HumanHT-12 V4.0). The data was analyzed and deposited to the GEO database (accession number: GSE79854).

All data analysis was performed using $\mathrm{R}$ (version 3.2.2) and $\mathrm{R}$ studio (Version 0.99.486). Raw Illumina transcriptomics data was normalized using quantile normalization (provided by normalize quantiles function from Bioconductor's preprocessCore package [Bolstad BM. preprocessCore: A collection of pre-processing functions. $\mathrm{R}$ package version 1.32.0.]) and was $\log 2$ transformed. Differential expression analysis of the normalized data was performed using the LIMMA package implemented in Bioconductor by employing an empirical Bayes $t$-test (Ritchie et al., 2015). Treatments with JNJ872, and infection with Udorn, or WSN were indicated in the design matrix for the linear fitting. The Benjamini-Hochberg method was used to adjust p-values for multiple hypothesis testing. Heatmap for transcriptomics data was generated using the pheatmap package (http://rpackages.ianhowson.com/cran/pheatmap/) based on $\log$ transformed expression data and hierarchical clustering of genes and samples in the heatmap was generated using the complete-linkage clustering method and Euclidian distances. 
A

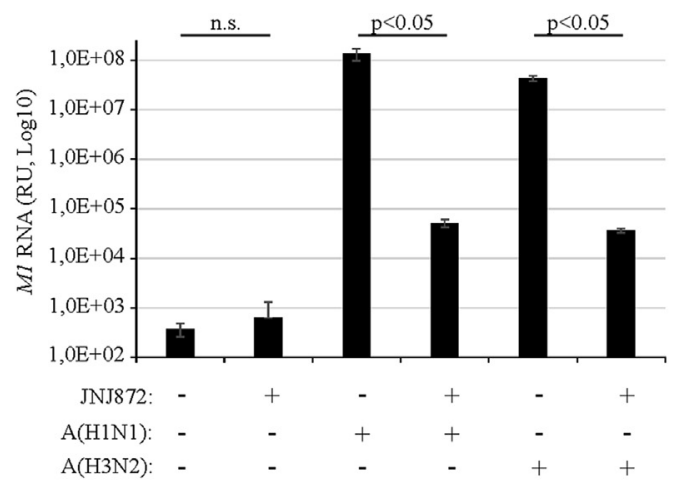

B

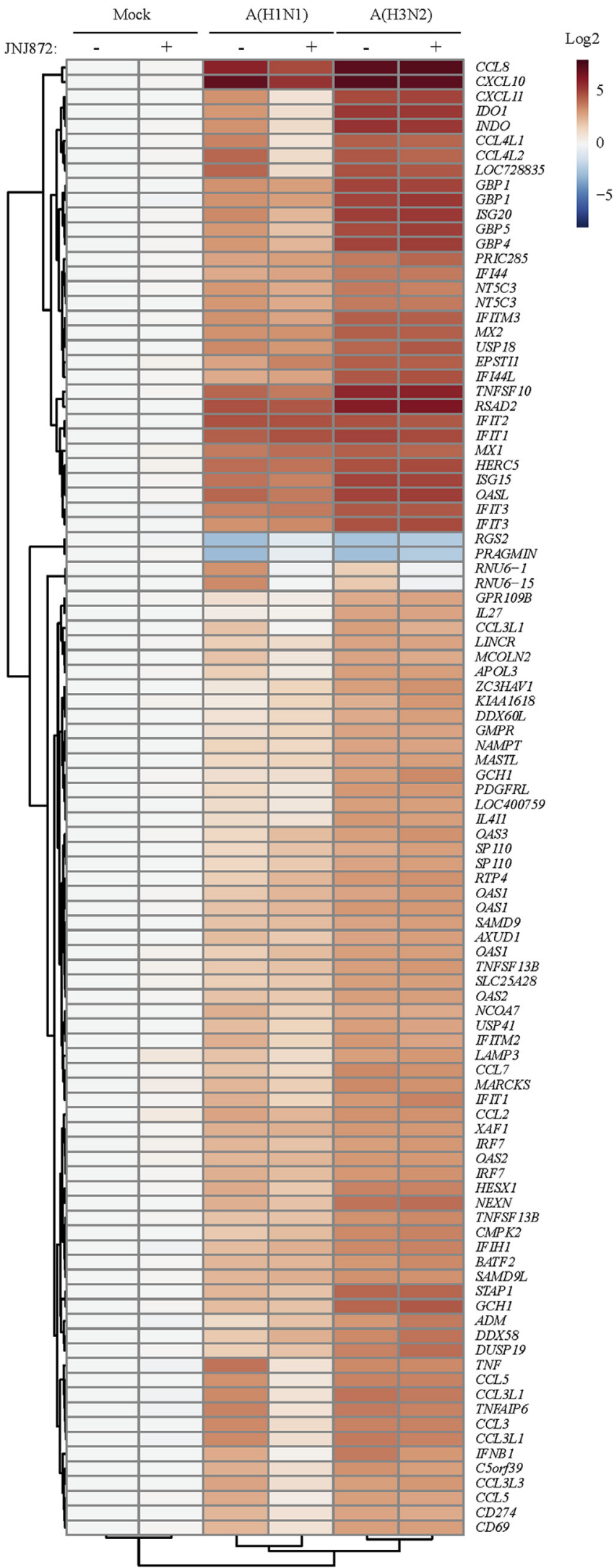

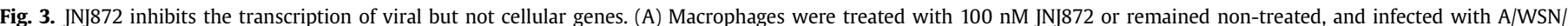

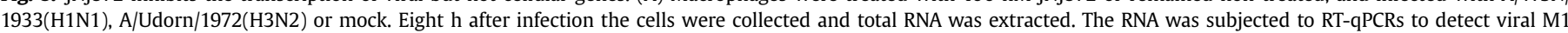

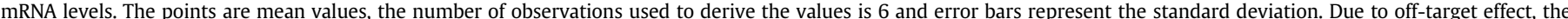

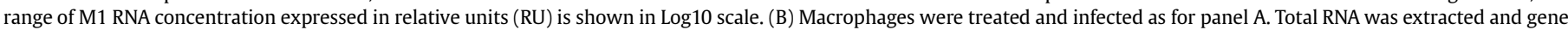

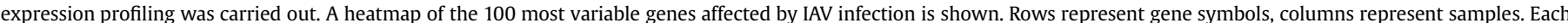

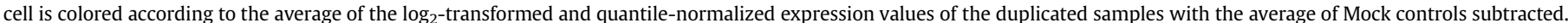
Complete-linkage hierarchical clustering based on Euclidian distances was used for clustering of genes and samples. 
A

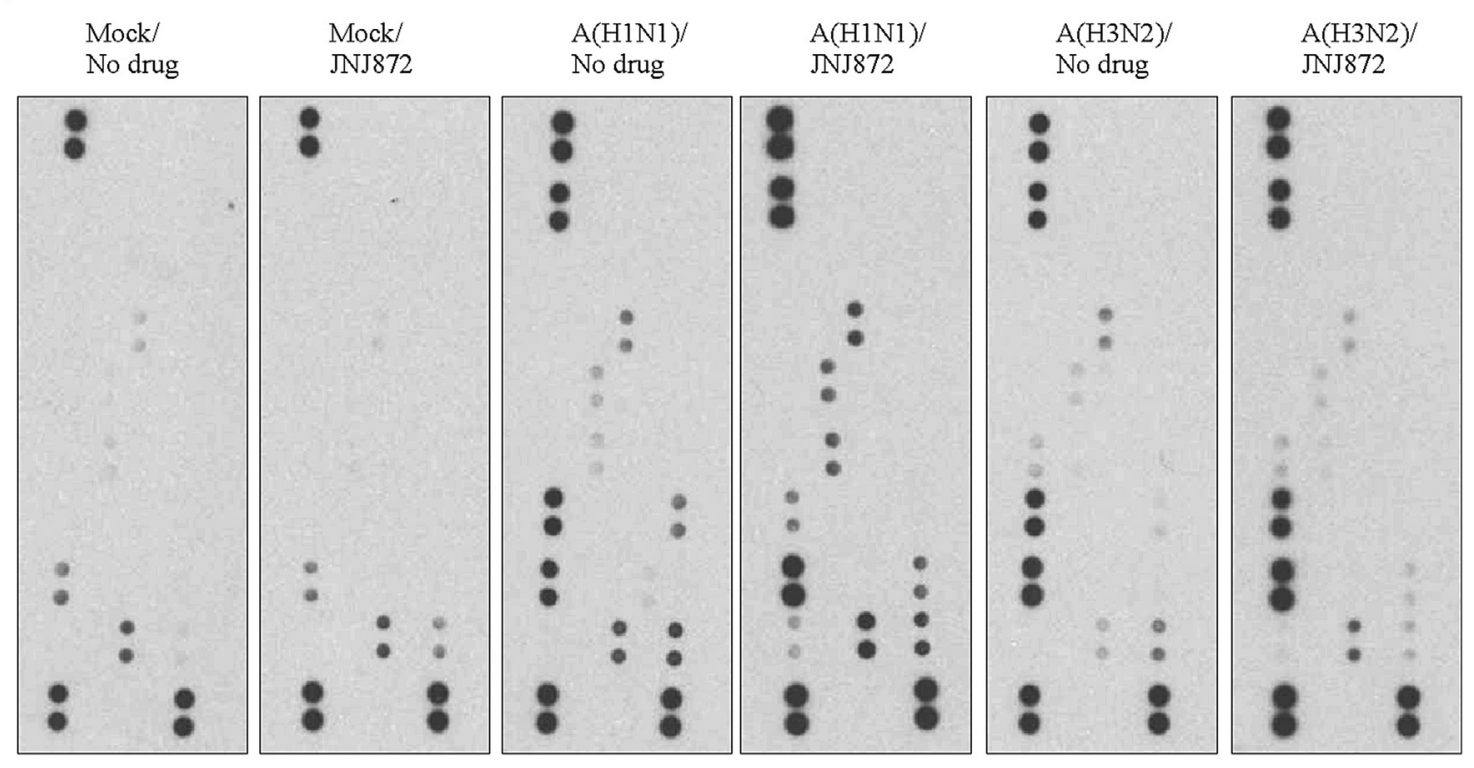

B

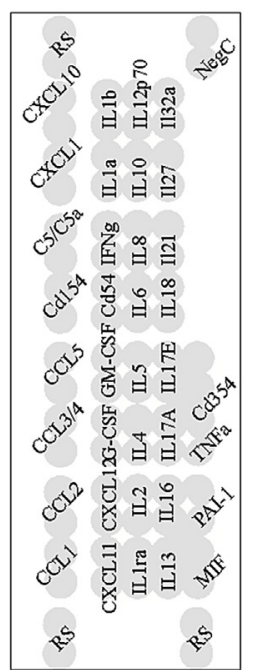

$\mathrm{C}$

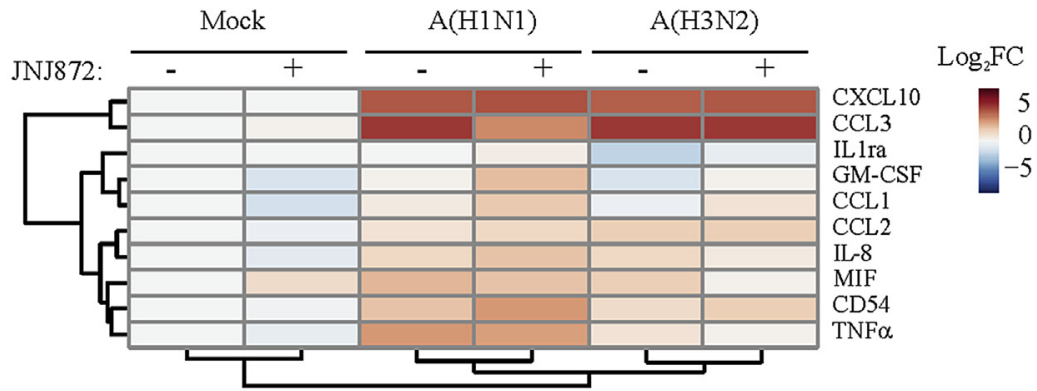

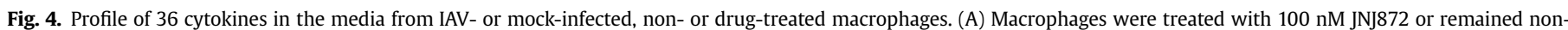

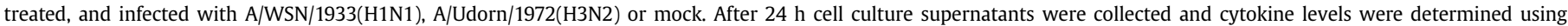

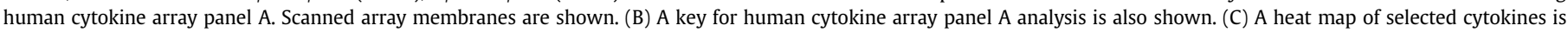

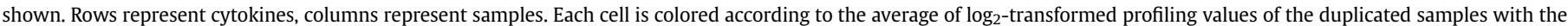
average of mock subtracted. Complete-linkage hierarchica clustering based on Euclidian distances was used for clustering of cytokines and samples.

\subsection{Cytokine profiling}

The medium from IAV- or mock-infected non- or drug-treated macrophages was collected at $24 \mathrm{~h}$ post infection and clarified by centrifugation for $5 \mathrm{~min}$ at $14,000 \mathrm{rpm}$. Cytokines were analyzed using Proteome Profiler Human Cytokine Array panel A kit or Proteome Profiler Human Cytokine Array Kit (R\&D Systems) according to manufacturer's instructions. The results were analyzed using Image software. Proteins with at least 2-fold difference in expression between mock and IAV-infected, JNJ872-treated or IAVinfected/JNJ872-treated samples were considered differentially expressed.

\subsection{Metabolomics}

Ten microliters of labeled internal standard mixture was added to $100 \mu \mathrm{L}$ of cell culture supernatant samples. Metabolites were extracted by adding 4 parts (1:4, sample:extraction solvent) of the $100 \%$ acetonitrile $+1 \%$ formic acid solvent. The collected extracts were dispensed in OstroTM 96-well plate (Waters Corporation, Milford, USA) and filtered by applying vacuum at a delta pressure of 300-400 mbar for $2.5 \mathrm{~min}$ on robot's vacuum station. Sample analysis was performed on an Acquity UPLC- system coupled to a triple quadrupole mass spectrometer (Waters Corporation, Milford, MA, USA). A detailed protocol and instrument parameters and list of measured metabolites were given elsewhere (Nikkanen et al., 2016; Roman-Garcia et al., 2014). Metabolomics data was log2 transformed for linear modeling and empirical-Bayes-moderated ttests using the LIMMA package (Smyth, 2004). To analyses the differences caused by the treatment of VX-787, a linear model was fit to each metabolite; treatments with JNJ872, and IAVs were indicated in the design matrix for this fit. The Benjamini-Hochberg 
method was used to correct for multiple testing. The significant metabolites were determined at a Benjamini-Hochberg false discovery rate (FDR) controlled at $10 \%$. The heatmap for all the metabolites was generated using the pheatmap package [Raivo Kolde (2015). pheatmap: Pretty Heatmaps. R package version 1.0.8.] based on $\log$ transformed profiling data.

\subsection{Primary and tertiary PB2 sequence analysis}

Sequences of PB2 proteins of human influenza A(H1N1)pdm09 and $\mathrm{A}(\mathrm{H} 3 \mathrm{~N} 2)$ viruses were downloaded from Influenza Virus Resource (http://www.ncbi.nlm.nih.gov/genomes/FLU/FLU.html) and from Global Initiative on Sharing Avian Influenza Data (http:// platform.gisaid.org/) databases. In total, we downloaded 4983 sequences of influenza $\mathrm{A}(\mathrm{H} 1 \mathrm{~N} 1) \mathrm{pdm} 09$ and 6385 sequences of influenza $A(H 3 N 2)$ viruses. Protein sequences were aligned using Muscle v3.8 and analyzed using Geneious 9 software (Edgar, 2004; Kearse et al., 2012). Similarity rates for each amino acid in alignments were calculated using Python 2.7 scripts. Structure of influenza PB2 cap-binding domain bound to JNJ872 was used for mapping JNJ872-interacting residues (Clark et al., 2014). Pymol was used for structure visualization (www.pymol.org).

\section{Results}

JNJ872 binds the cap-binding domain (CBD) of PB2. The X-ray structure of JNJ872 bound to the CBD of PB2 was solved (Clark et al., 2014). It revealed key interacting residues of PB2 (Fig. 1A). Our evolutionary analysis of $\mathrm{PB} 2$ sequences of 4987 influenza $\mathrm{A}(\mathrm{H} 1 \mathrm{~N} 1)$ pdm09 and $6385 \mathrm{~A}(\mathrm{H} 3 \mathrm{~N} 2)$ strains revealed that the JNJ872 interacting residues are highly conserved (Fig. $1 \mathrm{~B}$ and $\mathrm{C}$ ). This suggests that JNJ872 binds to a highly conserved site on the IAV PB2 protein.

The antiviral activity of JNJ872 was originally tested in MDCK cells against a broad range of IAV strains, including oseltamivir- and amantadine-resistant isolates as well as pandemic H1N1 and potentially-pandemic H5N1 strains (Byrn et al., 2015; Clark et al., 2014). We analyzed the cytotoxicity and antiviral activity of JNJ872 in human PBMC-derived macrophages infected with classical influenza A/WSN/1933(H1N1) or A/Udorn/307/1972 (H3N2) strains. First, we tested the effects of JNJ872 on the viability of noninfected and IAV-infected macrophages (multiplicity of infection (MOI 3); Fig. 2A). JNJ872 rescued macrophages from virusmediated death at non-cytotoxic concentrations 24 hpi. Second, we determined the $\mathrm{EC}_{50}$ for JNJ872. For this we pre-treated macrophages with increasing compound concentrations, infected them with the $A(\mathrm{H} 1 \mathrm{~N} 1)$ or $\mathrm{A}(\mathrm{H} 3 \mathrm{~N} 2)$ strain (at MOI 0.1$)$, collected the media 24 hpi and tittered viruses. The $\mathrm{EC}_{50}$ value for JNJ872 were 8 and $12 \mathrm{nM}$ for $\mathrm{A}(\mathrm{H} 1 \mathrm{~N} 1)$ and $\mathrm{A}(\mathrm{H} 3 \mathrm{~N} 2)$ strains, respectively, whereas the $\mathrm{CC}_{50}$ values were $>1 \mu \mathrm{M}$, giving selectivity indexes $(\mathrm{SI})>125$ and $>83$ for $\mathrm{A}(\mathrm{H} 1 \mathrm{~N} 1)$ and $\mathrm{A}(\mathrm{H} 3 \mathrm{~N} 2)$ strains, respectively (Fig. 2B). However, the $\mathrm{EC}_{50}$ and $\mathrm{SI}$ values varied depending on the macrophage preparation.

Next, we characterized the effect of JNJ872 on IAV-infected human macrophages in more detail. RT-qPCR analysis demonstrated that JNJ872 significantly attenuated the transcription of viral M1 RNA in macrophages, which were infected with $A(H 1 N 1)$ or $A(H 3 N 2)$ strains for $8 \mathrm{~h}$ (Fig. 3A). Transcriptomics analysis showed that JNJ872 allowed the expression of cellular type I and III IFNs and IFN-stimulated genes, such as C-C motif chemokine 2 (CCL2), CCL4, interleukine-8 (IL8), and C-X-C motif chemokine 10 (CXCL10) in IAV-infected macrophages (Fig. 3B). However, it did not allow the activation of IAV-mediated expression of CCL3, tumor necrosis factor (TNF) and several other genes to the same extent as observed in non-treated IAV-infected macrophages. In order to confirm our transcriptomics results, we analyzed a panel of pro- inflammatory cytokines in cell culture supernatants from IAVinfected human macrophages collected 24 hpi. In line with the transcriptomics results, the IAV-stimulated expression of CCL2, CCL4, IL8, MIF and CXCL10 by contrast to CCL3 and TNF $\alpha$, was unaffected by JNJ872 treatment (Fig. 4).

Our transcriptomics experiment also demonstrated that JNJ872 allowed some activation of IAV-mediated expression of several cellular genes, which are involved in tryptophan and nucleotide metabolism (such as indoleamine 2,3-dioxygenase 1 (IDO) and cytosolic 5'-nucleotidase 3A (NT5C3A)) (Fox et al., 2015; Meunier et al., 2016; Saha et al., 2010; Santos et al., 2014; Wang et al., 2016; Hu et al., 2011; Krapp et al., 2016; Pan et al., 2012; Schmidt et al., 2014; Wang et al., 2016; Zhu et al., 2013). To confirm this, we analyzed a panel of 110 polar metabolites in cell culture supernatants $24 \mathrm{hpi}$. We detected 70 metabolites (Fig. 5). The level of tryptophan was decreased and the level of its oxidation product Lkynurenin was elevated in both IAV-infected and IAV-infected/ JNJ872-treated macrophages, suggesting that JNJ872 did not affect the activity of IDO, an enzyme which catalyzes this reaction. Similarly, the level of adenosine was decreased, whereas the level of its catabolic products (adenine, inosine, inositol monophosphate

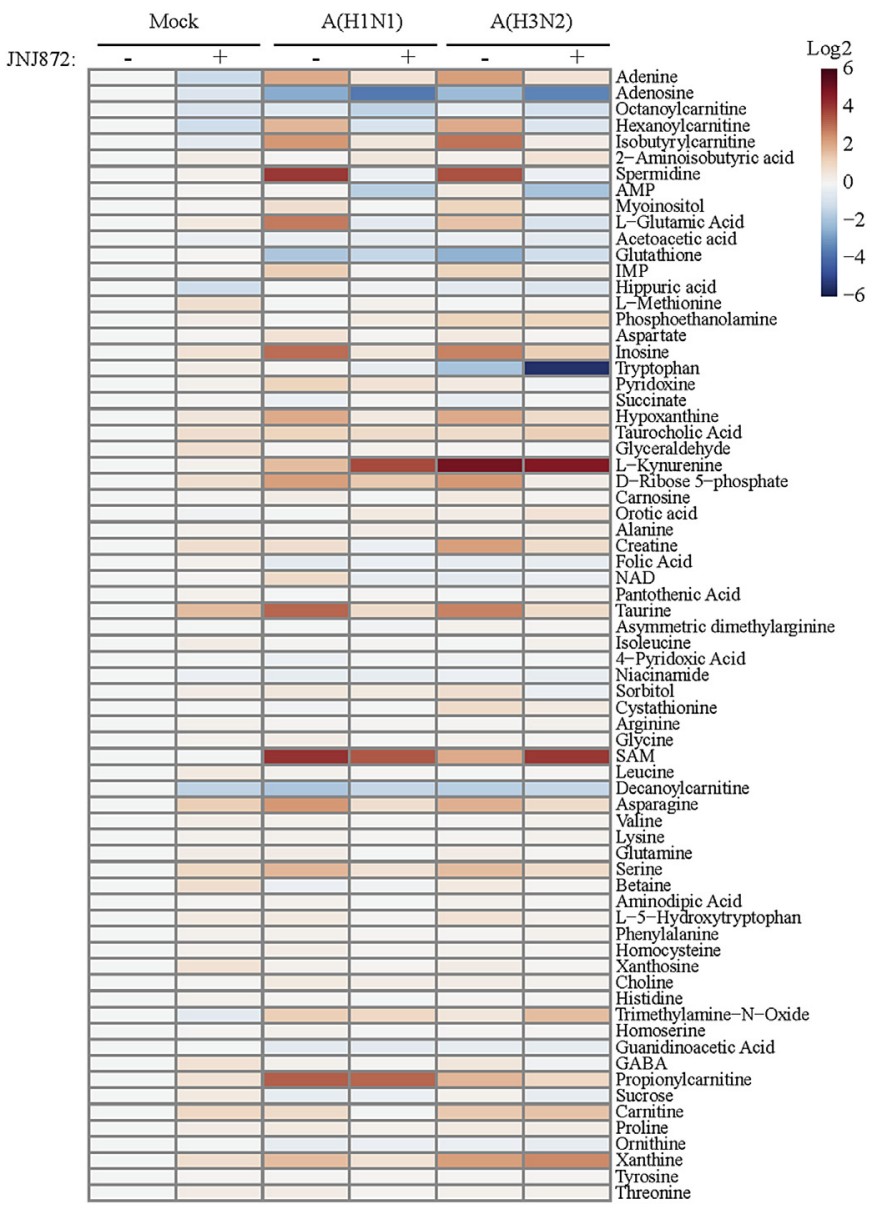

Fig. 5. Profile of 70 polar metabolites in the media of IAV- or mock-infected, non- or drug-treated macrophages. (A) Macrophages were treated with $100 \mathrm{nM}$ JNJ872 or remained non-treated, and infected with A/WSN/1933(H1N1), A/Udorn/1972(H3N2) or mock. After $24 \mathrm{~h}$ cell culture, supernatants were collected and metabolite levels were determined using mass spectrometry. A heat map of selected metabolites is shown. Rows represent metabolites, columns represent samples. Each cell is colored according to the average of $\log _{2}$-transferred profiling values of the duplicated samples with the average of Mock-infected, non-treated controls (i.e., the first column) subtracted. Metabolites are ranked based on the adjusted p-value. 
(IMP), and xanthine) were increased in both IAV-infected and IAVinfected/JNJ872 treated macrophages, suggesting that JNJ872 did not affect the activity of enzymes that catalyzed ribonucleotide degradation. Thus, JNJ872 possessed excellent anti-IAV but not immuno/metabolo-modulating effect.

It was shown that similarly to JNJ872, gemcitabine inhibits the transcription of IAV RNA, and allows the activation of cellular innate immune responses in infected macrophages (Soderholm et al., 2016). However, in contrast to JNJ872, gemcitabine targets cellular factors (Denisova et al., 2012). We tested whether the combination of virus-directed JNJ872 and cell-directed gemcitabine protects cells from IAV-mediated death at lower concentrations than that of the compounds alone. We found that a substantially lower JNJ872 concentration is needed to protect macrophages from IAV-mediated death when given with $100 \mathrm{nM}$ gemcitabine (Fig. 6). Thus, JNJ872 could be given in combination with non-cytotoxic concentration of gemcitabine to inhibit IAV infection.

\section{Discussion}

Oseltamivir, zanamivir, laninamivir, peramivir, amantadine, rimantadine, arbidol, and favipiravir (T-705) are approved for treatment of IAV infection in humans, whereas JNJ872, gemcitabine and many other IAV- and host-directed agents are under preclinical or clinical investigations (Loregian et al., 2014; Muller et al., 2012; Vanderlinden et al., 2014; Zumla et al., 2016). In the present study we demonstrated that JNJ872 targeted an evolutionary conserved site on the viral $\mathrm{PB} 2$ protein and efficiently attenuated influenza $\mathrm{A}(\mathrm{H} 1 \mathrm{~N} 1)$ and $\mathrm{A}(\mathrm{H} 3 \mathrm{~N} 2)$ infections with minimal impairment of host antiviral responses.

Mechanistically, treatment with JNJ872 inhibited the transcription of viral RNA in the nucleus of infected cells (Fig. S1). Meanwhile, the antiviral cascades were initiated by pattern recognition receptors (PRRs) in these cells. PRRs, such as TLR3, TLR7, MDA5, and RIG-I, recognized viral RNA in the cytoplasm and endosomes of infected cells and triggered NFkB- and IRF3mediated transcription of type I and III IFNs, which initiated expression of IFN-stimulated genes (ISGs), which encode cytokines and several enzymes (IDO, GBP1, GBP4, GBP5, NT5C3, CH25H, HERC5). It was shown that these enzymes mediated inflammation and restricted viral replication in infected cells (Fox et al., 2015; Gold et al., 2014; Kim et al., 2011; Nordmann et al., 2012; Tang et al., 2010; Wang et al., 2016). In particular, GBP1, GBP4, and GBP5, could play an active role in antiviral responses and inflammasome activation (Finethy et al., 2015; Friedman et al., 2016; Nordmann et al., 2012; Shenoy et al., 2012). In addition, these genes together with NT5C3A could catabolize ribonucleotides and, therefore, limit viral RNA transcription and replication (Fig. S1) (Fox et al., 2015; Meunier et al., 2016; Saha et al., 2010; Santos et al., 2014; Wang et al., 2016). IDO could also regulate inflammation and immune response since it catalyzes the production of Lkynurenine from tryptophan (Nguyen et al., 2010; Wang et al., 2010).

Our gene-expression results were supported by metabolomics analysis which also indicated that tryptophan and purine metabolic pathways were altered in response to IAV infection, and that JNJ872 treatment only slightly impaired them. These results indicated that JNJ872 treatment could limit viral replication and allowed the development of antiviral responses in IAV infected cells.

We also tested virus-directed JNJ872 in combination with celldirected gemcitabine. Gemcitabine is a nucleoside, ranking among the most prescribed anticancer drugs worldwide (Ciccolini et al., 2016). This nucleoside is converted into active triphosphorylated nucleotides interfering with DNA/RNA synthesis. In addition, gemcitabine possesses antiviral activity: it inhibits transcription and replication of IAV RNA, without impairment of antiviral responses (Denisova et al., 2012; Soderholm et al., 2016). We showed that the combination of JNJ872 and gemcitabine protected human macrophages from IAV-mediated death at substantially lower concentrations than that of the compounds alone.

We noticed that the EC50 values for JNJ872 varied depending on the macrophage preparations. The variabilities in EC50 values between macrophages derived from different blood donors could be associated with individual responses to infection. One can expect that certain variants in host genomic DNA or epigenetic modifications could affect antiviral responses and thereby facilitate or attenuate IAV replication, and, therefore, lead to different (individual) EC50 values for JNJ872 and its combinations with other drugs. Our results warrant further studies of JNJ872 and its combinations in cell cultures and animals, where these variations could be minimized. Moreover, studies in animal models could demonstrate not only the effect of these treatments on innate immune responses but also on adaptive responses. Therefore, JNJ872 alone or in combination with gemcitabine might mount resistance to infection with closely related IAV strains.

Altogether, our studies indicate that inhibiting the transcription and replication of viral RNA and perhaps later, but not earlier stages of IAV replication, could allow the development of efficient antiviral responses by infected cells. Such therapeutics could allow development of innate and acquired immunity, which would protect patients from re-infections with closely related IAV strains. However, the question remains whether severe IAV infections, which
A

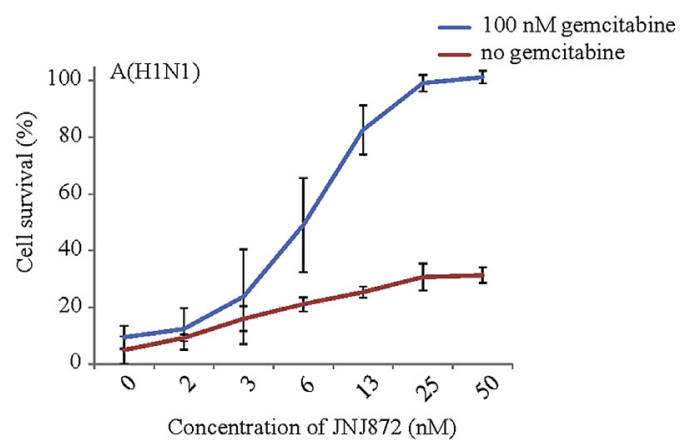

B

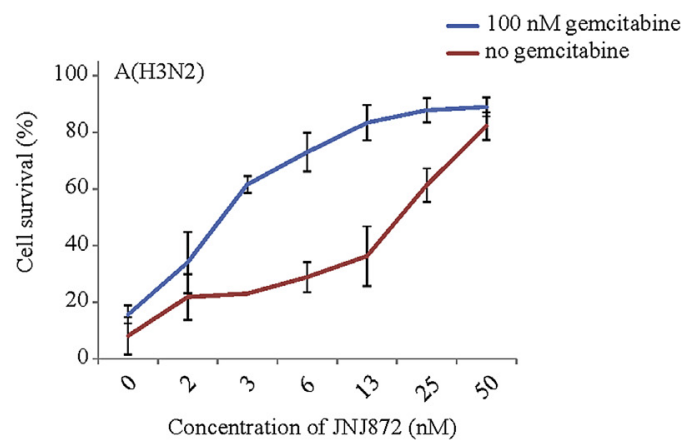

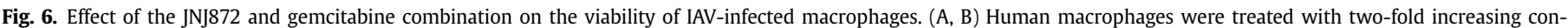

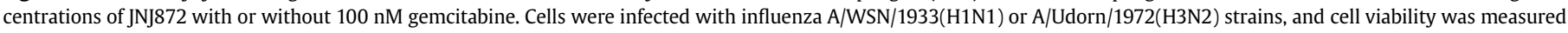
using a CTG assay $24 \mathrm{~h}$ after infection. All error bars, s.d. $(\mathrm{n}=3)$. 
are associated with cytokine storm, could be treated with such agents (Liu et al., 2016).

\section{Conclusion}

JNJ872 targets an evolutionary conserved site on influenza polymerase subunit PB2 and inhibits the transcription of viral genes. Moreover, a treatment with JNJ872 allowed the transcription of a set of cellular antiviral genes, and translation of cytokines and other antiviral proteins involved in the tryptophan or nucleotide metabolism. Furthermore, the combination of JNJ872 with gemcitabine protected human macrophages from IAV-mediated death at substantially lower concentrations than that of the compounds alone. VX-737 alone or in combination with other drugs, which inhibit IAV but allow development of antiviral responses, could be beneficial for treating IAV infected patients, because it would mount resistance to reinfections.

\section{Acknowledgments}

We thank Paivi Castren-Kortekangas and Jan Roelof Van Der Meer from Janssen Pharmaceuticals for providing JNJ872 and for the critical reading of the manuscript. We also thank Ilkka Julkunen for advice to use combination of JNJ872 and gemcitabine, and Roger Webster for influenza A/WSN/1933 reverse genetic systems. We gratefully acknowledge the authors, who submitted PB2 sequences to the GISAID and IVR databases, on which this research is based. This study was supported by Jane and Aatos Erkko foundation, University of Helsinki (three-year research grant No. 465/51/2014) and CIMO foundation (grant No. TM-15-9787).

\section{Appendix A. Supplementary data}

Supplementary data related to this article can be found at http:// dx.doi.org/10.1016/j.antiviral.2016.07.008.

\section{References}

Anastasina, M., et al., 2015. The C terminus of NS1 protein of influenza A/WSN/ 1933(H1N1) virus modulates antiviral responses in infected human macrophages and mice. J. Gen. Virol. 96, 2086-2091. http://dx.doi.org/10.1099/ vir.0.000171.

Belanov, S.S., et al., 2015. Genome-wide analysis of evolutionary markers of human influenza $\mathrm{A}(\mathrm{H} 1 \mathrm{~N} 1) \mathrm{pdm} 09$ and $\mathrm{A}(\mathrm{H} 3 \mathrm{~N} 2)$ viruses may guide selection of vaccine strain candidates. Genome Biol. Evol. 7, 3472-3483. http://dx.doi.org/10.1093/ gbe/evv240.

Byrn, R.A., et al., 2015. Preclinical activity of VX-787, a first-in-class, orally bioavailable inhibitor of the influenza virus polymerase PB2 subunit. Antimicrob. Agents Chemother. 59, 1569-1582. http://dx.doi.org/10.1128/ AAC.04623-14.

Ciccolini, J., et al., 2016. Pharmacokinetics and pharmacogenetics of Gemcitabine as a mainstay in adult and pediatric oncology: an EORTC-PAMM perspective. Cancer Chemother. Pharmacol. http://dx.doi.org/10.1007/s00280-016-3003-0.

Clark, M.P., et al., 2014. Discovery of a novel, first-in-class, orally bioavailable azaindole inhibitor (VX-787) of influenza PB2. J. Med. Chem. 57, 6668-6678. http://dx.doi.org/10.1021/jm5007275.

Denisova, O.V., et al., 2012. Obatoclax, saliphenylhalamide, and gemcitabine inhibit influenza a virus infection. J. Biol. Chem. 287, 35324-35332. http://dx.doi.org/ 10.1074/jbc.M112.392142.

Denisova, O.V., et al., 2014. Akt inhibitor MK2206 prevents influenza pH1N1 virus infection in vitro. Antimicrob. Agents Chemother. 58, 3689-3696. http:// dx.doi.org/10.1128/AAC.02798-13.

Edgar, R.C., 2004. MUSCLE: a multiple sequence alignment method with reduced time and space complexity. BMC Bioinforma. 5 (113) http://dx.doi.org/10.1186/ 1471-2105-5-113.

Edinger, T.O., et al., 2014. Entry of influenza A virus: host factors and antiviral targets. J. Gen. Virol. 95, 263-277. http://dx.doi.org/10.1099/vir.0.059477-0.

Finethy, R., et al., 2015. Guanylate binding proteins enable rapid activation of canonical and noncanonical inflammasomes in Chlamydia-infected macrophages. Infect. Immun. 83, 4740-4749. http://dx.doi.org/10.1128/IAI.00856-15.

Fox, J.M., et al., 2015. Interferon lambda upregulates IDO1 expression in respiratory epithelial cells after influenza virus infection. J. Interferon Cytokine Res. 35, 554-562. http://dx.doi.org/10.1089/jir.2014.0052.
Friedman, K., et al., 2016. Medullary carcinoma of the colon: a distinct morphology reveals a distinctive immunoregulatory microenvironment. Mod. Pathol. http:// dx.doi.org/10.1038/modpathol.2016.54.

Gold, E.S., et al., 2014. 25-Hydroxycholesterol acts as an amplifier of inflammatory signaling. Proc. Natl. Acad. Sci. U. S. A. 111, 10666-10671. http://dx.doi.org/ 10.1073/pnas.1404271111.

Hoffmann, E., et al., 2000. A DNA transfection system for generation of influenza A virus from eight plasmids. Proc. Natl. Acad. Sci. U. S. A. 97, 6108-6113. http:// dx.doi.org/10.1073/pnas.100133697.

$\mathrm{Hu}$, Y., et al., 2011. Guanylate binding protein 4 negatively regulates virus-induced type I IFN and antiviral response by targeting IFN regulatory factor 7 . J. Immunol. 187, 6456-6462. http://dx.doi.org/10.4049/jimmunol.1003691.

Kearse, M., et al., 2012. Geneious Basic: an integrated and extendable desktop software platform for the organization and analysis of sequence data. Bioinformatics 28, 1647-1649. http://dx.doi.org/10.1093/bioinformatics/bts199.

Kim, B.H., et al., 2011. A family of IFN-gamma-inducible 65-kD GTPases protects against bacterial infection. Science 332, 717-721. http://dx.doi.org/10.1126/ science.1201711.

Krapp, C., et al., 2016. Guanylate binding protein (GBP) 5 is an interferon-inducible inhibitor of HIV-1 infectivity. Cell Host Microbe 19, 504-514. http://dx.doi.org/ 10.1016/j.chom.2016.02.019.

Li, T.C., et al., 2015. Clinical implications of antiviral resistance in influenza. Viruses 7, 4929-4944. http://dx.doi.org/10.3390/v7092850.

Liu, Q., et al., 2016. The cytokine storm of severe influenza and development of immunomodulatory therapy. Cell Mol. Immunol. 13, 3-10. http://dx.doi.org/ 10.1038/cmi.2015.74.

Loregian, A., et al., 2014. Antiviral strategies against influenza virus: towards new therapeutic approaches. Cell Mol. Life Sci. 71, 3659-3683. http://dx.doi.org/ 10.1007/s00018-014-1615-2.

Meunier, E., et al., 2016. Interferon-inducible GTPases in cell autonomous and innate immunity. Cell Microbiol. 18, 168-180. http://dx.doi.org/10.1111/cmi.12546.

Muller, K.H., et al., 2012. Emerging cellular targets for influenza antiviral agents. Trends Pharmacol. Sci. 33, 89-99. http://dx.doi.org/10.1016/j.tips.2011.10.004.

Nguyen, N.T., et al., 2010. Aryl hydrocarbon receptor negatively regulates dendritic cell immunogenicity via a kynurenine-dependent mechanism. Proc. Natl. Acad. Sci. U. S. A. 107, 19961-19966. http://dx.doi.org/10.1073/pnas.1014465107.

Nikkanen, J., et al., 2016. Mitochondrial DNA replication defects disturb cellular dNTP pools and remodel one-carbon metabolism. Cell Metab. 23, 635-648. http://dx.doi.org/10.1016/j.cmet.2016.01.019.

Nordmann, A., et al., 2012. A new splice variant of the human guanylate-binding protein 3 mediates anti-influenza activity through inhibition of viral transcription and replication. FASEB J. 26, 1290-1300. http://dx.doi.org/10.1096/ fj.11-189886.

Ohman, T., et al., 2010. Cytosolic RNA recognition pathway activates 14-3-3 protein mediated signaling and caspase-dependent disruption of cytokeratin network in human keratinocytes. J. Proteome Res. 9, 1549-1564. http://dx.doi.org/ $10.1021 /$ pr901040u.

Pan, W., et al., 2012. Guanylate-binding protein 1 participates in cellular antiviral response to dengue virus. Virol. J. 9 (292) http://dx.doi.org/10.1186/1743-422X9-292.

Pirhonen, J., Sareneva, T., Kurimoto, M., Julkunen, I., Matikainen, S., 1999. Virus infection activates IL-1 beta and IL-18 production in human macrophages by a caspase-1-dependent pathway. J. Immunol. 162 (12), 7322-7329.

Ritchie, M.E., et al., 2015. Limma powers differential expression analyses for RNAsequencing and microarray studies. Nucleic Acids Res. 43, e47. http:// dx.doi.org/10.1093/nar/gkv007.

Roman-Garcia, P., et al., 2014. Vitamin $\mathrm{B}(1)(2)$-dependent taurine synthesis regulates growth and bone mass. J. Clin. Invest 124, 2988-3002. http://dx.doi.org/ 10.1172/JCI72606.

Saha, B., et al., 2010. Gene modulation and immunoregulatory roles of interferon gamma. Cytokine 50, 1-14. http://dx.doi.org/10.1016/j.cyto.2009.11.021.

Santos, A., et al., 2014. Pyrimidine-5'-nucleotidase Campinas, a new mutation (p.R56G) in the NT5C3 gene associated with pyrimidine-5'-nucleotidase type I deficiency and influence of Gilbert's Syndrome on clinical expression. Blood Cells Mol. Dis. 53, 246-252. http://dx.doi.org/10.1016/j.bcmd.2014.05.009.

Schmidt, S.V., et al., 2014. New insights into IDO biology in bacterial and viral infections. Front. Immunol. 5 (384) http://dx.doi.org/10.3389/fimmu.2014.00384.

Shenoy, A.R., et al., 2012. GBP5 promotes NLRP3 inflammasome assembly and immunity in mammals. Science 336, 481-485. http://dx.doi.org/10.1126/ science.1217141.

Smyth, G.K., 2004. Linear models and empirical bayes methods for assessing differential expression in microarray experiments. Stat. Appl. Genet. Mol. Biol. 3 http://dx.doi.org/10.2202/1544-6115.1027. Article3.

Soderholm, S., et al., 2016. Immuno-modulating properties of saliphenylhalamide, SNS-032, obatoclax, and gemcitabine. Antivir. Res. 126, 69-80. http:/ dx.doi.org/10.1016/j.antiviral.2015.12.011.

Tang, Y., et al., 2010. Herc5 attenuates influenza A virus by catalyzing ISGylation of viral NS1 protein. J. Immunol. 184, 5777-5790. http://dx.doi.org/10.4049/ jimmunol.0903588.

Vanderlinden, E., et al., 2014. Emerging antiviral strategies to interfere with influenza virus entry. Med. Res. Rev. 34, 301-339. http://dx.doi.org/10.1002/ med.21289.

Wang, X., et al., 2016. RNA interference screening of interferon-stimulated genes with antiviral activities against classical swine fever virus using a reporter virus. Antivir. Res. 128, 49-56. http://dx.doi.org/10.1016/j.antiviral.2016.02.001. 
Wang, Y., et al., 2010. Kynurenine is an endothelium-derived relaxing factor produced during inflammation. Nat. Med. 16, 279-285. http://dx.doi.org/10.1038 nm.2092.

Zhu, Z., et al., 2013. Nonstructural protein 1 of influenza A virus interacts with human guanylate-binding protein 1 to antagonize antiviral activity. PLoS One 8 e55920. http://dx.doi.org/10.1371/journal.pone.0055920.

Zumla, A., et al., 2016. Host-directed therapies for infectious diseases: current status, recent progress, and future prospects. Lancet Infect. Dis. 16, e47-63. http:// dx.doi.org/10.1016/S1473-3099(16)00078-5. 